\title{
2D OCEAN WAVES SPECTRA FROM SPACE: A CHALLENGE FOR VALIDATION \& SYNERGETIC USE
}

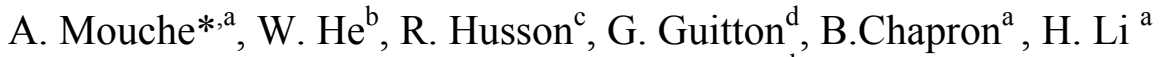 \\ ${ }^{a}$ IFREMER/LOPS Pointe du Diable, 29280 Plouzané, France; ' NOTC, 60, Xianyang Road, Nankai, \\ Tianjin, China; ${ }^{\mathrm{C}} \mathrm{CLS}$, Avenue La Pérouse, Bâtiment le Ponant, 29280 Plouzané, France; \\ ${ }^{\mathrm{d} O c e a n D a t a L a b}$, Deolen 29280 Locmaria-Plouzané, France.
}

\begin{abstract}
Sentinel-1 A now routinely acquires data over the ocean since 2014. Data are processed by ESA through the Payload Data Ground Segment up to Level-2 for Copernicus users. Level-2 products consist of geo-located geophysical parameters related to wind, waves and ocean current. In particular, Sentinel-1A wave measurements provide 2D ocean swell spectra (2D wave energy distribution as a function of wavelength and direction) as well as integrated parameters such as significant wave height, dominant wavelength and direction for each partition. In 2016, Sentinel-1 B will be launched by ESA and GF-3 by CNSA. Then in 2018, CFOSAT (China France Oceanography Satellite project), a joint mission from the Chinese and French Space Agencies, will be launched. They will also provide 2D Ocean waves spectra. This paper focuses on the techniques used to validate 2D-ocean waves as measured by satellite and the challenges and opportunities of such a program for ocean waves measurements from space.
\end{abstract}

Keywords: Ocean remote sensing, synthetics aperture radar, ocean wave-spectrometer, 2D ocean wave spectrum, Level4.

\section{INTRODUCTION}

Since April 2014, Sentinel-1 A is routinely measuring 2D ocean swell spectra over open-ocean. Except from north east Atlantic, the coverage is global. Sentinel-1 B will have the same capacities. Its launched is announced for April 2016. GF-3, a high resolution C-band SAR should be launched in 2016 by the CNSA. In the line of ENVISAT/ASAR, GF-3 will carry on board a SAR with a Wave Mode to measure 2D ocean swell spectra over open-ocean at global scale. Then the French and Chinese space agencies have planned a joint mission called CFOSAT to launch a wave-spectrometer SWIM in 2018. Given the expected life duration of these missions, we should be in position of getting 2D ocean waves measurements (level-2 products) from 4 different satellites in 2018. This would be a world first.

As for other geophysical variables such as ocean surface wind or sea surface temperature, we would like to take benefit of the diversity of these existing waves measurements in order to provide a homogeneous and qualified dataset at the highest resolution in both time and space. The merge and/or analysis of lower-level data into a new product is defined as a level-4 product. To date such product from ocean waves spectra measurements does not exist. In fact, we have never been in the position of having ocean waves spectra from different space missions at the same time. An example of 2Docean wave spectrum as measured by Sentinel-1 A the $8^{\text {th }}$ of March 2016 at midnight and modeled by Wave Watch 3 model are presented on Figure 2 (a) and (b). Corresponding SAR image is presented on Figure 2 (c).

However, to be properly merged, measurements have to be consistent and the limitations of each sensor well understood and taken into account. As a consequence, the validation of each mission and the inter-calibration between the different sensors represents two mandatory steps prior any data combination.

This paper focuses on the techniques used to validate 2D-ocean waves as measured by satellite and the opportunities of having such a tremendous number of wave measurements at global scale for ocean studies. Section 2 deals with the calibration and the inter-calibration methodologies. Section 3 describes the data and the corresponding parameters to validate and the reference data. Section 4 presents the concept of waves measurements merging to gather all available wave measurements at the storm-scale. Sections 5 presents applications regarding validation and wave studies based on Sentinel-1 A data.

Remote Sensing of the Oceans and Inland Waters: Techniques, Applications, and Challenges, edited by Robert J. Frouin, Satheesh C. Shenoi, K. H. Rao, Proc. of SPIE Vol. 9878, 98780L (c) 2016 SPIE · CCC code: 0277-786X/16/\$18 · doi: 10.1117/12.2227178 


\section{VALIDATION \& INTER-CALIBRATION}
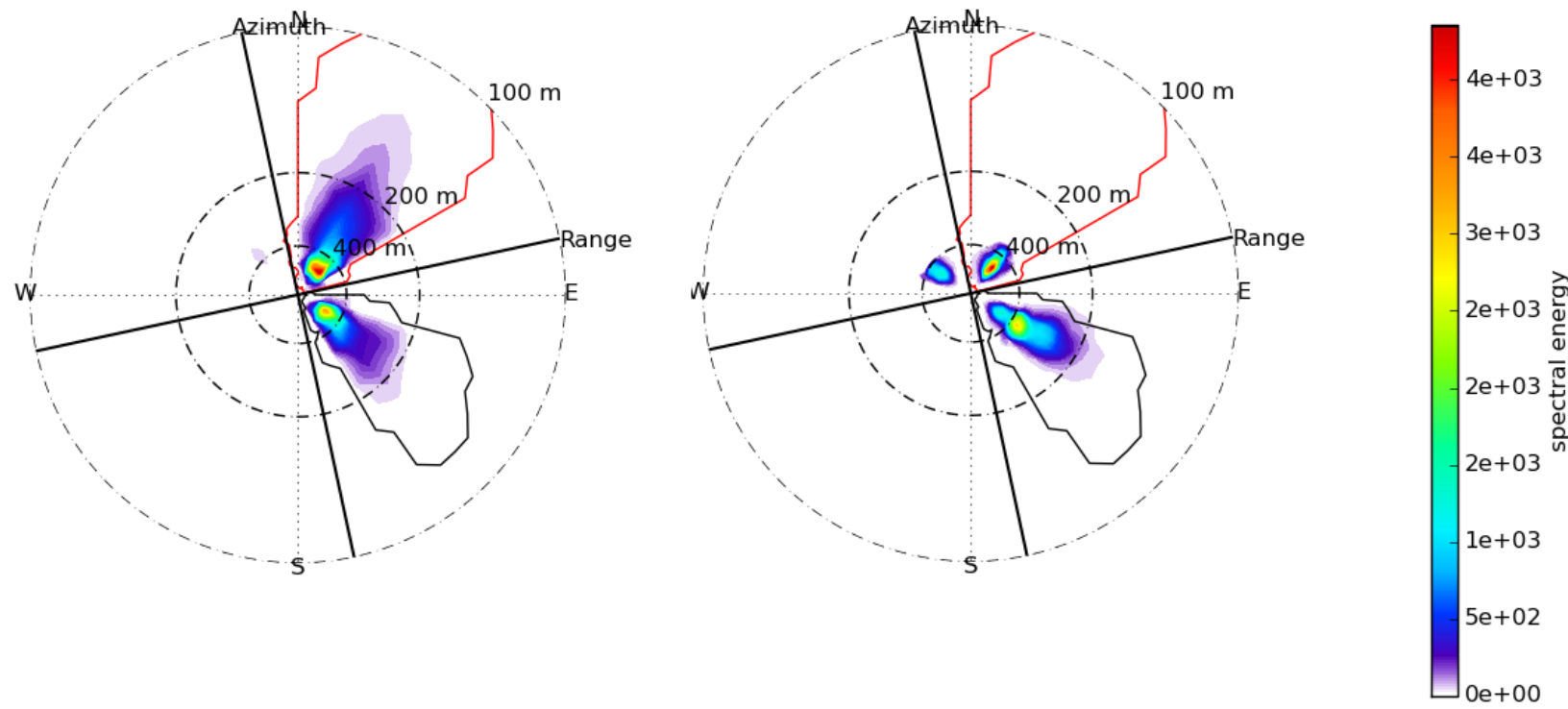

(a)

(b)

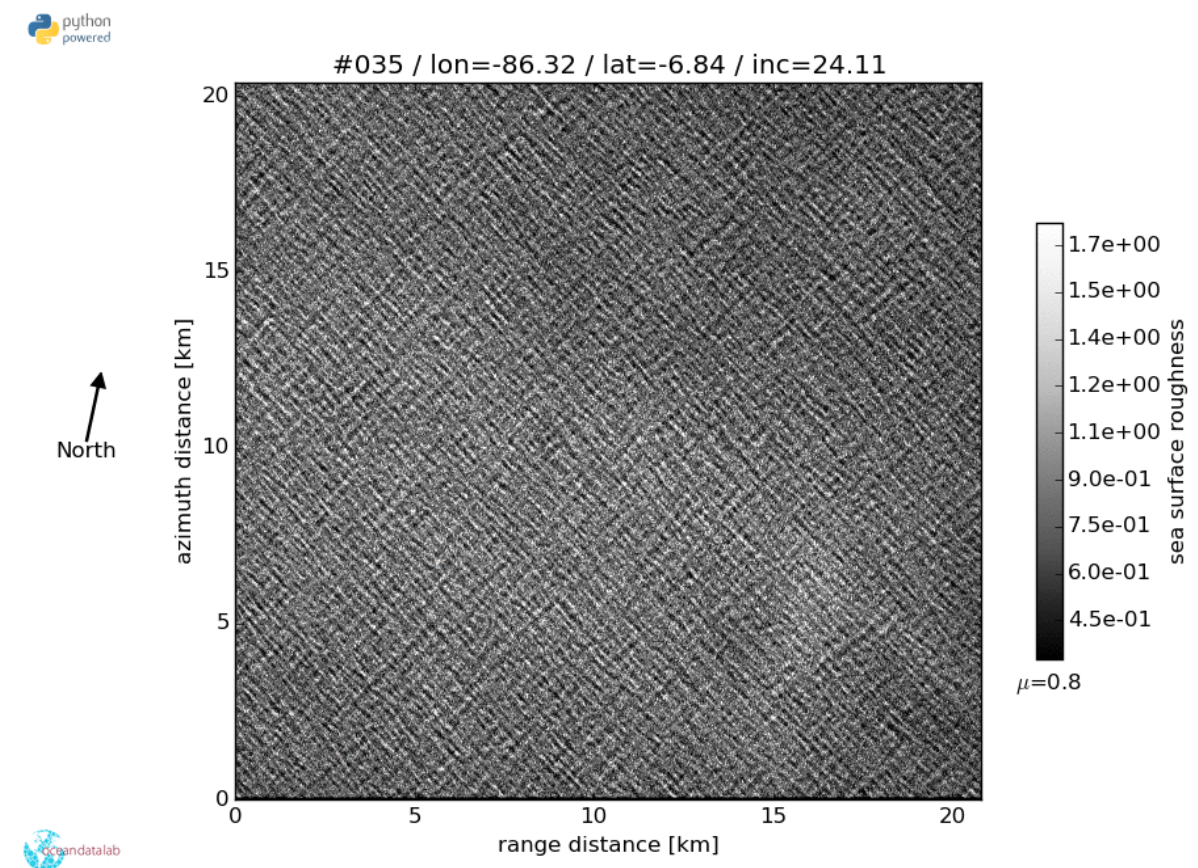

(c)

Illustration 1: (a) Wave Watch $32 D$ ocean wave spectrum. (b) Co-located Example of 2D ocean wave spectrum measured by Sentinel$1 \mathrm{~A}$. The limits of the partitions for the 2 most energetic wave systems are derived from the partitioning of WW3 spectrum. They are indicated in color and plotted on the two wave spectra. Co-location is less than $1.5 \mathrm{~h}$ and less than $0.25^{\circ}$.(c) Corresponding SAR image is presented. Modulation of the intensity are the swell signature. The crossing lines are due to the two crossing swell systems. 
To take benefit of each mission assets, a careful validation and inter-calibration of each level-2 parameters to be combined for level-4 product have to be performed. This has to be done for each mission and for each sensor with respect to its limitations. For instance, Synthetic Aperture Radar on board Sentinel-1 is only able to measure ocean swells between $1000 \mathrm{~m}$ (thanks to the large coverage) and $200 \mathrm{~m}$ (depending on the wind) with a high resolution in azimuth direction. In the contrary, SWIM is expected to get waves propagation direction with a resolution of $15^{\circ}$ and a $180^{\circ}$ ambiguity but to measure ocean waves in a range of wavelength between $70 \mathrm{~m}$ and $500 \mathrm{~m}$. The main characteristics of each sensors to be used for Level-4 are detailed in section 3.

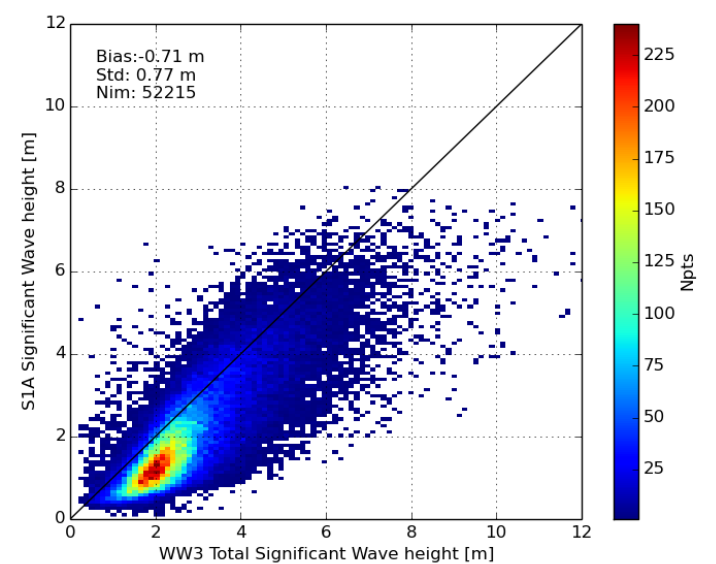

(a)

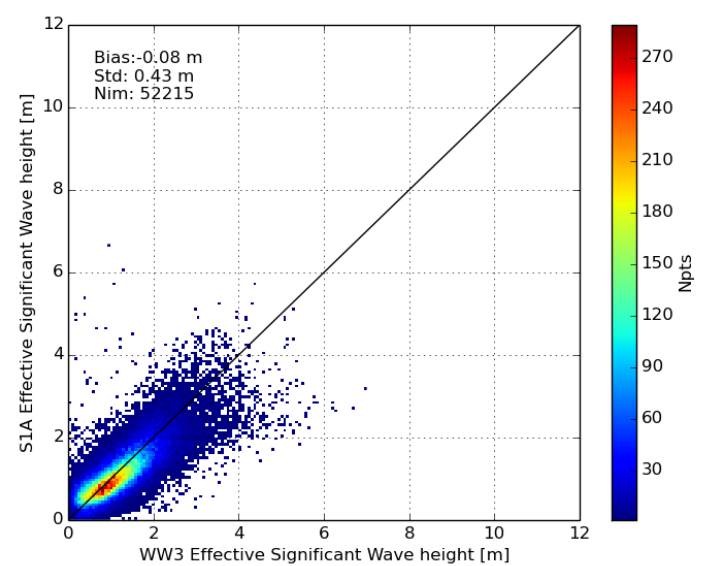

(b)

Illustration 2: Comparisons of Significant Wave Height from Wave Watch 3 and Sentinel-1 A. All data acquired from January to March 2016 are considered here. (a) Total significant wave height as modeled by WW3 and the significant wave height from Sentinel-1 A are compared. The full range of available wave number is considered. (b) Effective significant wave height has been computed for both the model and Sentinel-1 based on Sentinel-1 A performances.

\section{Validation \& Inter-calibration}

Validation: For a given mission, a given acquisition mode and all ocean waves parameters, the validation consists in assessing its performances against a reference dataset. For all missions, acquisition modes and ocean wave parameters, the same reference dataset has to be used. Here, in-situ buoys, altimeters measurements as well as model outputs are used as reference data. In addition, dedicated validation campaigns can be set up (This will be the case for CFOSAT for instance). They can represent a valuable source of information for validation.

Inter-calibration: Before merging the measurements obtained from different sensors or different acquisition modes, the consistency between the different datasets need to be evaluated. To this aim, situations where the sensors, despite their different properties (measurements techniques but also orbit characteristics), are expected to measure the same wave systems are compared and inter-calibrated.

\section{Ocean waves parameters}

For each mission, the 2D ocean waves spectrum is the main geophysical quantity included in the level-2 products delivered by the space agencies. The 2D ocean wave height spectrum is defined as the density spectrum of wave heights as a function of the 2D wavenumber vector $\mathbf{k}$ (expressed hereafter in polar coordinates $(k, \phi)$ ). In addition, we also derive the integrated parameters describing the different detected wave systems from these spectra. They are the basis of the combined analysis and product we propose in section 4. 
At a given time and location, several wave systems can co-exist. These situations are called mixed seas or crossed seas. Each wave system (also called partition) is defined on a bounded domain in $k$ and $\phi$. Each partition is obtained after the partitioning step of the 2D-spectrum. For instance, in the case presented on Figure 1 (c), we can clearly see in the image intensity the combination of two modulations due to crossing swells. The two swell systems can also be seen in the 2D ocean waves spectra from the model or the data. Partitions are indicated in color and reveal two orthogonal swell systems with wavelength larger than 400 $\mathrm{m}$.

Each wave system can be described by 3 parameters estimated on the bounded domain: (i) the significant wave height, (ii) the peak wave period and (iii) the peak wavelength. They are the main parameters to validate. Other parameters such as the spectral width of the peak can be defined from the 2D ocean wave spectrum. They are not considered here.

The total significant wave height is defined by:

$$
H_{s}=4 \sqrt{\iint_{k} F(k, \phi) k d k d \phi},
$$

where $k$ is the wavenumber, $\phi$ the direction and $F(k, \phi)$ the ocean wave height spectrum. We also introduce the concept of effective significant wave height. It is defined by:

$$
H_{s_{\text {eff }}}=4 \sqrt{\int_{k_{\text {eff }}} \int_{\phi} F(k, \phi) k d k d \phi},
$$

where $k_{\text {eff }}$ indicates the common range of wavelengths where the two dataset can be compared. Indeed, for each instrument, the integration domain in $k$ may be different because the detected wavelengths are not necessarily the same between sensors. The effective significant wave height is used to compare two sources that do not have the same performances. The integration domain must be selected to intersects the wavelengths domain covered by the two sensors. For SAR, the range of wavelength to consider is $\left[0, k_{a z c}\right]$ where $k_{a z c}$ is the wave number associated to the cut-off. This threshold is estimated from SAR products during the processing and strongly related to the sea state conditions [1,2].

Figure 2 (a) presents an example of comparison between the total significant wave height as modeled by WW3 and the significant wave height from Sentinel-1 A obtained when doing the integration over the whole range of wavelengths (Eq. (1)) without taking care of SAR limitations. As expected, results are not consistent as the ocean wave spectrum from SAR is not expected to be geophysical for wavelength below the azimuth cut-off. In the contrary, on figure 2 (b), where the effective significant wave height (Eq. (2)) has been computed for both WW3 and Sentinel-1 A, they are in good agreement, with a bias around 0 and a standard deviation around $50 \mathrm{~cm}$.

Finally, integrated parameters for each partition are defined by:

$$
H_{s}^{\text {part }}=4 \sqrt{\int_{k_{\text {part }}} \int_{\phi_{\text {part }}} F(k, \phi) k d k d \phi,}
$$

where $k_{\text {eff }}$ and $\phi_{\text {eff }}$ define the wavelength domain on which the partition is defined. This is the significant wave height of the partition.

$\lambda_{\text {peak }}^{\text {part }}=\frac{2 \pi}{k_{\text {peak }}^{\text {part }}}$ defines the peak wavelength of the partition. $k_{\text {peak }}^{\text {part }}$ is the wavenumber at the peak of the 1-D ocean

wave spectrum $F^{\text {part }}(k)$ defined as $F^{\text {part }}(k) \int_{\phi_{\text {part }}} F(k, \phi) d \phi$. 
The peak direction of the partition is the direction at the peak of the directional spectra. The directional spectrum $D^{\text {part }}(\phi)$ is computed from the wave spectra as $D^{\text {part }}(\phi)=\int_{k \text { part }} F(k, \phi) k d k$,

The peak direction is then found as $\phi_{\text {peak }}^{\text {part }}=\max (D(\phi))$.

For instance, on Figure 1 (a), we can see that in this particularly case, Wave Watch 3 model predicts two swell systems with orthogonal directions. They are also observed by the SAR as shown by the 2D-ocean wave spectrum available in Level-2 Sentinel 1 A product. Note that we can observed this crossing swell in the modulation of the image intensity. Spectral domains related to the two partitions as predicted by the model are indicated in red and black on both ocean wave spectra. In this case we can see a good agreement between model and measurement.

\section{Static \& Dynamic co-location}

Before being compared, two measurements need to be co-located in space and time within a certain spatio-temporal window to be determined. For ocean swell measurements obtained in open-ocean data are considered co-located when they are distant by less than $100 \mathrm{~km}$ and acquired in a time window of 3 hours max. There are several types of colocations possible when dealing with ocean wave validation and inter-calibration in open ocean. They are detailed below and illustrated in the Figure 3.

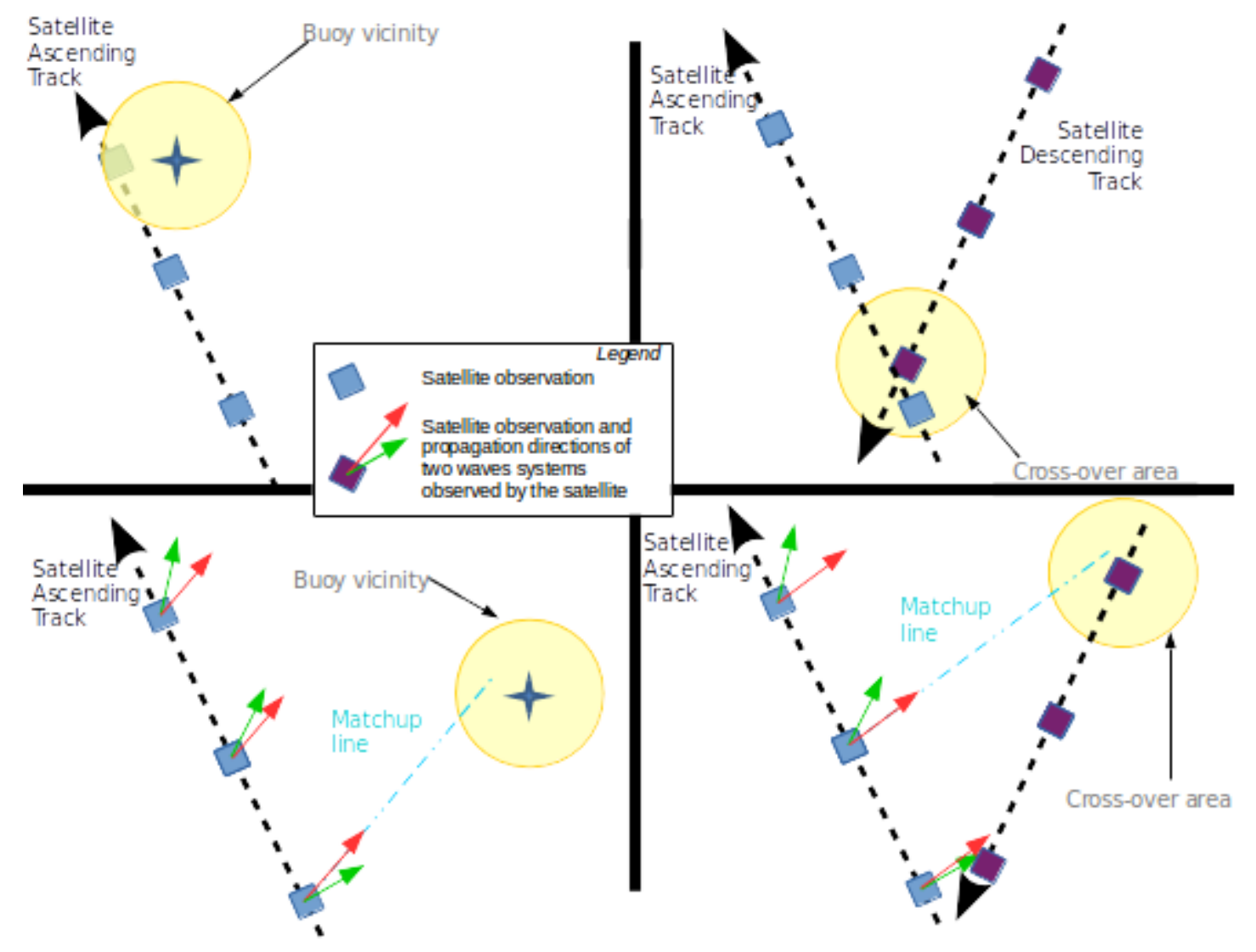

Illustration 3: Different types of co-locations. Top panel left: Static co-locations. Top panel right: Static cross-over. Bottom panel left: Dynamic co-location. Bottom panel right: Dynamic cross-over. 
Static co-locations: The measurement to validate or inter-calibrate has to be acquired in the vicinity of the reference data location and within a given time frame. This is illustrated on Figure 3 (top-left).

Satellite-satellite co-locations: It is the cross-over technique. it is inherited from Altimetry. It consists in selecting intersecting fields of view from different satellites within a certain spatio-temporal window. The wave systems as measured by the different sensors are then compared. The concept is illustrated on Figure 3 (top-right). This method has been used by [3] to show the inconsistency between swell measurements from ENVISAT/ASAR as obtained with descending and ascending tracks.

Dynamic co-locations consist in comparing swell systems acquired at different locations in space and time. For a given observation $\mathrm{O}_{1}$, the ocean wave spectrum is partitioned into wave systems. Swell systems from $\mathrm{O}_{1}$ are then propagated with respect to time (backward or forward) up to another fixed location where an ocean wave measurement $\left(\mathrm{O}_{2}\right)$ is available. The propagation is done assuming the dispersion relationship in open ocean without any impact of ocean surface currents and the dissipation relationship proposed by [4]. Dynamic co-location concept is illustrated on Figure 3 (bottom-left). This method allows an increase of match-ups at buoys that can be located in areas where no acquisitions are done.

Dynamic cross-over is like dynamic co-location but the two observations $\mathrm{O}_{1}$ and $\mathrm{O}_{2}$ are moving. This is illustrated on Figure 3 (bottom-right). This will be used for inter-comparisons between different satellites.

\section{SATELLITE \& REFERENCE DATA}

\section{Sentinel-1 A \& B (satellite data)}

Sentinel-1 A \& B are part of a 4 earth observation satellite series for Europe's Copernicus environmental program. Sentinel-1 A has been launched in April 2014. Sentinel-1 B launch is scheduled for April 2016. Sentinel-1 satellites carry C-Band $(5.405 \mathrm{GHz})$ SAR sensors and have a dedicated acquisitions mode (Wave Mode or WV) for ocean swell measurements. WV is single polarization only ( $\mathrm{HH}$ or $\mathrm{VV}$ ) and acquires alternatively on near range (incidence angle: $23.5 \mathrm{deg}$; also called WV1) and far range (incidence angle: $36.5 \mathrm{deg}$; also called WV2). Wave mode acquires data in 20 $\mathrm{km}$ by $20 \mathrm{~km}$ vignettes every $100 \mathrm{~km}$ along the orbit. Vignettes on the same incidence angle are separated by $200 \mathrm{~km}$. Swaths alternate incidence angles between near range and far range $\left(23^{\circ}\right.$ and $36.5^{\circ}$ respectively). Table below recalls the main characteristics for the 3 sensors to be combined.

\section{SWIM/CFOSAT (satellite data)}

COFSAT launch is scheduled for mid-2018 the latter. It will carry Ku-Band Radar: the wave scatterometer (SWIM) and the wind scatterometer (SCAT). SWIM (Surface Wave Investigation and Monitoring) is a near-nadir (0, 2, 4, 6, 8 and $10^{\circ}$ incidence angles) Ku-Band azimuthally scanning real-aperture radar designed for measuring the directional spectrum of ocean waves. The acquisition sequence is defined by a macro-cycle. A cycle is the consecutive time spent at a given incidence and the macro-cycle is the elementary set of cycles, which combines all incidences. The nominal macro-cycle is $\left\{0^{\circ}, 2^{\circ}, 4^{\circ}, 6^{\circ}, 8^{\circ}, 10^{\circ}\right\} .6^{\circ}, 8^{\circ}$ and $10^{\circ}$ are the incidence angles dedicated to the $2 \mathrm{D}$ ocean waves spectrum measurement. The global swath of the instrument is about $90 \mathrm{~km}$ left and right of the nadir track. Wave directional spectra will be delivered at a scale of $70 \times 90 \mathrm{~km}$ on each side of the track for wavelength range $70 \mathrm{~m} \sim 500 \mathrm{~m}$. Targeted accuracy are $10 \%$ on wavelength, $15^{\circ}$ on direction (with however a $180^{\circ}$ ambiguity in direction) and about 15 to $20 \%$ on energy in the energy containing part of the spectrum. Table below recalls the main characteristics for the 3 sensors to be combined. The reader can also refer to [5] for details regarding the CFOSAT mission.

\section{GF-3 (satellite data)}

As part of high-resolution earth observing system of China, GF-3 satellite will be launched in September 2016. It will carry high-resolution C-band (5.4GHz) SAR sensor. One of its acquisition modes will be Wave Mode (WV) dedicated for global ocean swell measurements. GF-3 wave mode will collect SAR data for quad-polarization in $5 \mathrm{~km}$ by $5 \mathrm{~km}$ 
vignettes every $50 \mathrm{~km}$ along the satellite orbit. The default incident angle for GF- $3 \mathrm{WM}$ will be set to be 23 degrees, and switchable between 20 and $41 \mathrm{deg}$. It is expected to detect the swell with the wavelength $150 \sim 500 \mathrm{~m}$.

\begin{tabular}{|l|c|c|c|c|c|c|}
\hline & $\begin{array}{c}\text { Frequency } \\
{[\mathbf{G H z}]}\end{array}$ & $\begin{array}{c}\text { Incidence } \\
\text { angles [deg] }\end{array}$ & $\begin{array}{c}\text { Azimuth and } \\
\text { range Res [m] }\end{array}$ & $\begin{array}{c}\text { Product } \\
\text { resolution } \\
{[\mathbf{k m}]}\end{array}$ & $\begin{array}{c}\text { Radar } \\
\text { Aperture Type }\end{array}$ & $\begin{array}{c}\text { Ocean surface } \\
\text { wavelength } \\
{[\mathbf{m}]}\end{array}$ \\
\hline Sentinel-1 & 5.405 & $23.5 ; 36.5$ & $3 \times 5$ & $\begin{array}{c}20 \times 20 \\
\text { every } 100 \mathrm{~km}\end{array}$ & Synthetic & {$[200-1000]$} \\
\hline SWIM & 13.575 & $6 ; 8 ; 10$ & $0.94 ; 1.41 ; 1.41$ & $70 \times 90$ & Real & {$[70-500]$} \\
\hline GF-3 & 5.4 & 23.0 & 10 & $\begin{array}{c}5 \times 5 \text { every } 50 \\
\mathrm{~km}\end{array}$ & Synthetic & {$[150-500]$} \\
\hline
\end{tabular}

\section{Existing in-situ buoy network (reference data)}

Satellite missions considered here are dedicated to 2D ocean wave measurements in open ocean. As a consequence, algorithms used for ocean waves measurements from these missions are not developed for coastal areas, where bathymetry can dramatically modify the waves properties (diffraction, refraction, wave-current interactions) such as significant wave height, wavelength and wave direction. In some cases (Sentinel-1 for instance), there is even no coastal acquisition in the modes dedicated to ocean wave measurements.

In addition, most of existing buoys are located in Europe or North America, mostly in coastal areas and do not necessarily measure the full $2 \mathrm{D}$ ocean spectrum. As a consequence, they are very few 2D ocean wave measurements that can be directly used as reference data. Initiated with the ESA GlobWave project, IFREMER now routinely downloads, archives, qualifies, harmonizes and formats in-situ wave measurements from various networks worldwide (e.g. CDIP, NDBC, ...). When properly done, comparisons with buoys remain meaningful. They are possible when satellite observations are not too far from the buoys or for particular orientations of incoming swell systems. For instance, ocean swells measurements from ENVISAT/ASAR in Wave Mode have been validated against buoys [4]. Finally, some buoys such as Stratus (NDBC 32012) are very well located in open ocean and can be used for our activities. Figure 7 (right panel) illustrates a typical comparison between stratus buoy and Sentinel-1 A effective significant wave height measurements.

\section{Altimeter (reference data)}

Altimeters offer a unique opportunity to get total significant wave height measurements in open ocean. As explained above, each mission measures ocean waves in a particular range of wavelengths and does not measure the total significant wave height. This is particularly true for SAR that focuses mainly on swell. However, a systematic comparison is a good indicator to monitor the performances of the instrument with respect to time during the mission duration. Figure 5 (b) illustrates a comparison between significant wave heights for swell from Sentinel-1 A and the total significant wave height from Altika/SARAL altimeter. As expected the total significant wave height from the altimeter is larger than the significant wave height from the SAR. Here the comparison is done over one month of data acquired at global scale in December 2015. For SWIM, as ocean waves are expected to be measured for wavelengths between $70 \mathrm{~m}$ and $500 \mathrm{~m}$, direct comparisons with altimeters should be much more consistent.

\section{Wave Model (reference data)}

The ability of wave models to produce 2D ocean wave spectra for each satellite measurement makes them a unique tool to validate separately each wave system after partitioning of the $2 \mathrm{D}$ ocean wave spectrum. It also enables to get quickly a dataset representative of a large range of situations (wind, waves, rain, ...) to work with. This is particularly interesting at the beginning of the mission when few data are available. However, triple co-locations between model, satellite data and 
another reference data (buoy or altimeter) are mandatory to check the model performances. IFREMER uses Wave Watch 3 and sets up a system to provide a model output for all Sentinel-1 acquisitions. Figure 5 (a) shows an example of comparisons between WW3and SARAL/Altika from a triple co-location with Sentinel-1 A for December 2015.

\section{LEVEL-4 OCEAN WAVES PRODUCT}

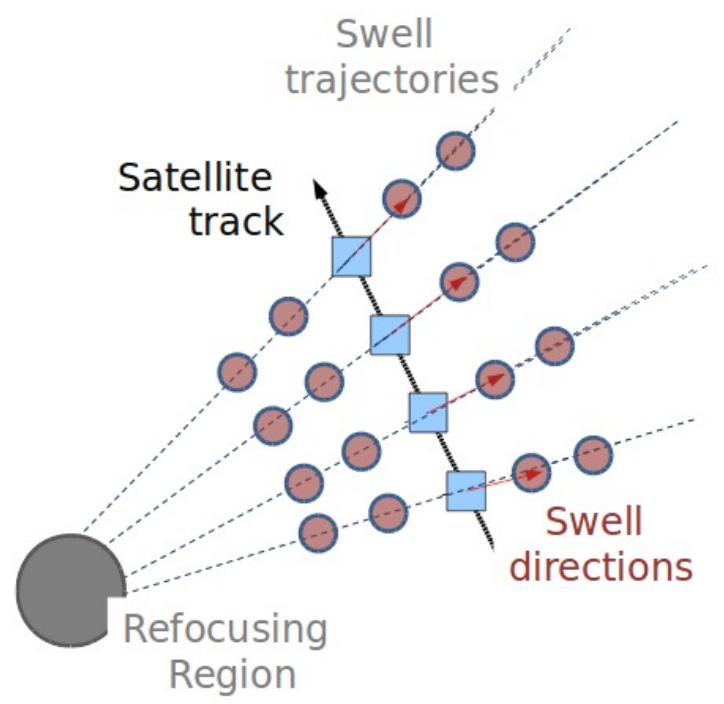

Illustration 4: Fireworks concept. Blue squares stand for satellite ocean swell measurements. Red dots indicate the location of the swell during its propagation backward toward the refocusing region (large grey circle to represent the storm) and forward toward the coasts.

Anticipating the large number of available data and the specificity of each sensor, the Level-4 product intends to describe ocean waves at global scale by taking benefit of each mission assets. For instance, Synthetic Aperture Radar on board Sentinel-1 is only able to describe ocean swell typically between $1000 \mathrm{~m}$ (thanks to the large coverage) and $200 \mathrm{~m}$ (depending on the wind) with a high resolution in direction. In the contrary, SWIM is expected to get waves propagation direction with a resolution of $15^{\circ}$ and a $180^{\circ}$ ambiguity but to measure ocean waves in a range of wavelength between 70 $\mathrm{m}$ and $500 \mathrm{~m}$.

As defined here, Level-4 wave product aims at gathering together all the wave measurements originating from the same storm. It will provide the main integrated parameters (wavelength, direction, period and significant wave height) of ocean waves and of its associated storm (location, duration, intensity).

The concept (called Fireworks) has already been applied to ENVISAT/ASAR data [4]. It is presented on Figure 4. A detailed description of the steps can be found in [3]. Such kind of product strongly relies on the pioneer works by Munk and Snodgrass [7,8] but has been adapted to satellite observations. We recall the main steps to make such a product below:

- Observations collection and filtering: Wave systems parameters are estimated from 2D spectra coming from various sources. Observations are filtered with respect to their quality. Observations are blue squares on Figure 4. Red arrows stand for swell directions as obtained from observations and used for propagation.

- Propagation: Observations and its associated integrated parameters are then propagated in space and time, backward. On Figure 4, red dots indicate the location of the swell during its propagation backward toward the refocusing region. Dashed lines indicate the trajectory. Propagation is done according to the wave direction given by the observation at its group velocity given by: 
$c_{g}=\frac{1}{2} \sqrt{\frac{g \lambda}{2 \pi}}$, where $\lambda$ is the wavelength of the wave partition to propagate and $g$ the acceleration of gravity constant.

We assume deep ocean and no wave-current interactions during the propagation. Wind-wave interactions are also neglected. As a consequence, if at a certain time and location the wind speed is too high with respect to the phase speed of the wave we propagate, then the propagation is stopped. Phase speed is given by:

$c=\frac{g T}{2 \pi}$, where $\mathrm{T}$ is the period of the wave partition to propagate. In open ocean, $\lambda=\frac{g}{2 \pi} T^{2}$.

Propagated swell locations at different propagation times are red circles on Figure 4. Trajectories are in dotted lines.

- Refocusing: Back-propagated swell observations are analyzed to determine the storm source location in both time and space. For the storm source description, the back-propagated waves analysis can also be complemented by wind measurements analysis. Refocusing area is large grey disk on Figure 4.

- Significant wave Height: As already mentioned, outside of island shadows and in the absence of ocean currents, swell propagates at group velocity and along geodesics in deep water. Under this assumption only phenomena modifying the swell energy during the propagation are (i) are angular spreading, acting in transverse direction, and frequency dispersion, acting in radial direction and (ii) dissipation. These two effects on the energy decay have been recently revisited by [4] and [6]. Based on these works we model the swell dissipation far from the storm source $(\mathrm{d}>4000 \mathrm{~km})$ by:

$$
H_{\text {swell }}(\alpha)=H_{\text {swell }}\left(\alpha_{0}\right) \sqrt{\frac{\alpha_{0} \sin \left(\alpha_{0}\right)}{\alpha \sin (\alpha)}} \exp \left(-\mu \frac{\alpha-\alpha_{0}}{2}\right) \text {, }
$$

where $\alpha$ is any angular great-circle distance from the storm source and $\alpha_{0}$ a angular great-circle distance from the storm source associated to an observation where the significant wave height of the swell has been measured. $\mu$ is a constant.

- Consistency of data at storm scale: Observations than could not be associated to a storm are filtered out. For a given storm, the consistency of its associated waves observations with respect to significant wave height distribution is checked. Outliers are filtered out.

Swell parameters associated to qualified observations and propagations are the main components of the product. The time sampling of the product is expected to be regular (at least $6 \mathrm{~h}$ ). The space sampling is not regular and fully driven by observations and propagated observations.

Note that the data that have been filtered out can contain valuable information. For instance, in the particular case of CFOSAT/SWIM, as the minimum detected wavelength will be smaller than with other sensors (up to $70 \mathrm{~m}$ ) we may reject waves that has been affected by current during their propagation. Wave-current interactions is still a subject for research. As a consequence, the Level-4 method proposed here could also serve to map the area where the waves propagation is affected by current.

\section{APPLICATIONS}

\section{Sentinel-1 A validation}

First, we perform a validation of Wave Watch 3 against altimeter. Results are presented on Figure 5 (a). In this case, we did a triple co-location between Sentinel-1 A measurements, Wave Watch 3 model outputs and Altika measurements acquired in December 2015. Bias is found around $0 \mathrm{~m}$ and standard deviation $35 \mathrm{~cm}$. These good results on average explain why the model can be used to perform a statistical validation of ocean wave products measured from SAR. 
Indeed, Level-2 ocean products from Sentinel-1 A are targeting a bias lower than $10 \mathrm{~cm}$ with a standard deviation lower than $50 \mathrm{~cm}$ for the significant wave height parameter. Figure 5 (b) shows an example of co-location between significant wave height computed from Sentinel-1 A 2D-ocean wave spectra and measured by altimeter. We clearly observe that the significant wave height estimated from Sentinel-1 A is not a total significant wave height. However, such kind of systematic comparisons can help to monitor the performances of the sensor and detect possible issues.

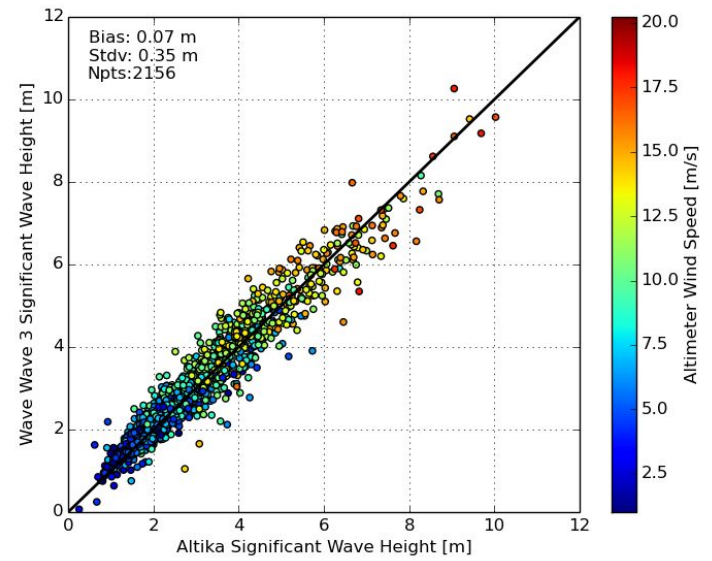

(a)

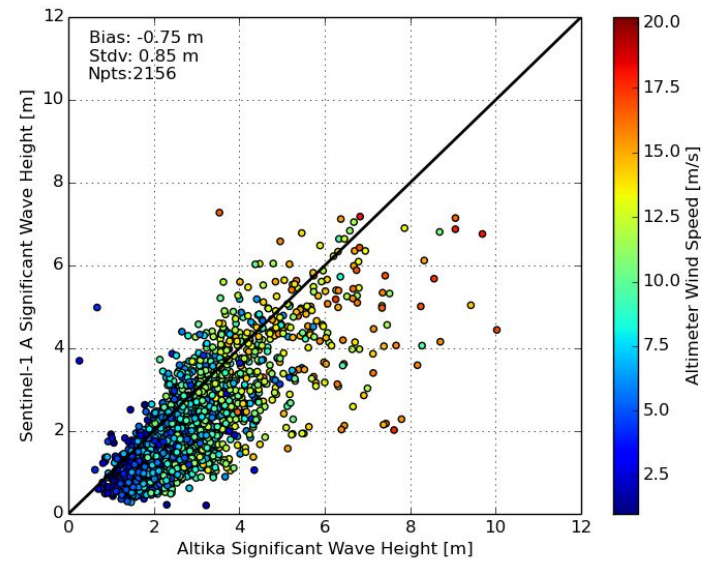

(b)

Illustration 5: Left: (a) Comparisons between total significant wave height measured Altimeter and predicted by Wave Watch 3 model. (b) Comparisons between total significant wave height measured Altimeter and significant wave height by Sentinel-1 A. Co-locations are less than $1.5 \mathrm{~h}$ and less than $0.25^{\circ}$.

Then, Sentinel-1 A validation is performed against Wave Watch 3 model. A ocean wave spectrum is produced for each Sentinel-1 acquisition in Wave Mode. This validation is done at global scale and performed every day for both WV1 and WV2 and for both ascending and descending passes. To avoid contamination by iceberg or sea ice, an arbitrary filtering is set to consider only latitude lower than $60^{\circ}$. A monthly report is produced. As SAR imaging mechanism does not allow the measurement of ocean waves below the azimuth cut-off [2], we use the effective significant wave height evaluated for waves larger than the azimuth cut-off (see Eq. (2)). For Wave Mode 2, the mean bias is around $0 \mathrm{~m}$ and deviation standard is less than $50 \mathrm{~cm}$. As observed on Figure 2 (b), for the first three months of 2016, we obtained a bias of $-8 \mathrm{~cm}$ and a standard deviation of $43 \mathrm{~cm}$. We also use this co-located dataset to compare the performances per wave system. Results are presented on Figure 6 . Bias is found to be $-27 \mathrm{~cm}$ and deviation standard $77 \mathrm{~cm}$. We also observe that the performances depends on wind direction with respect to the antenna look angle (see Figure 6 (b)) and the wavelength (see Figure 6 (c)). These results indicate that improvements can certainly be proposed to refine the algorithm used by ESA to process Level-1 data into Level-2 Ocean product. This topic is not addressed in this paper.

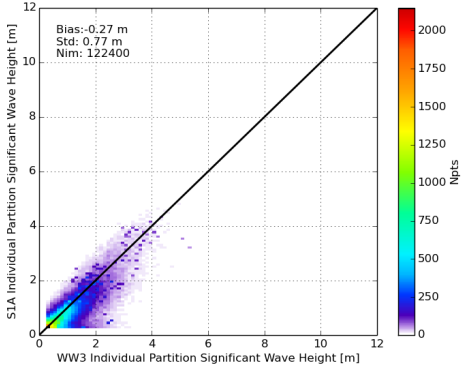

(a)

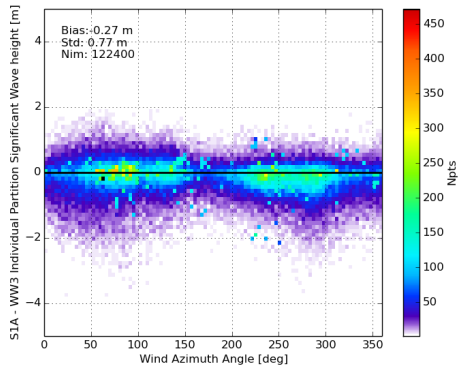

(b)

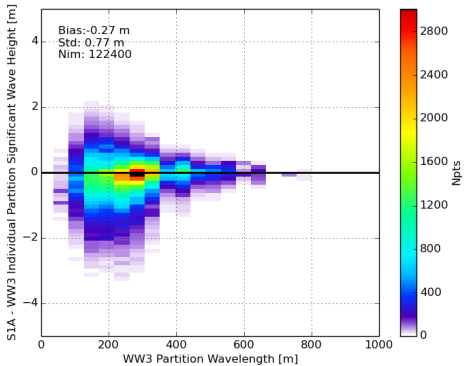

(c)

Illustration 6: (a) Comparisons between effective significant wave heights measured by Sentinel-1 A and modeled by Wave Watch 3 for each partition. (b) \& (c) Bias is presented respectively as a function of wind direction relative to the antenna and wavelength. Colocation is less than $1.5 \mathrm{~h}$ and less than $0.25^{\circ}$. 
We also performed preliminary comparisons of the effective significant wave height against NDBC Stratus buoy (station 32012) measurements. Indeed, this buoys is located in the south Pacific ocean in deep water. Location is $19.819 \mathrm{~S}$ 84.737 W. Since December 2015, we can see on Figure 7 (left panel) that we have more than 8 co-locations per month. This led us to a total of 39 co-locations. Here we consider a co-location valid when the distance between satellite swath center and buoy location is less than $100 \mathrm{~km}$. We compare (see Figure 7 right panel) the effective significant wave height with the wavelength limit such that we consider wavelengths above the azimuth cut-off as estimated from the SAR measurements. Bias is almost $0 \mathrm{~m}$ and standard deviation around $40 \mathrm{~cm}$. To date, this number of co-locations is too low and the geophysical situations are not representative of a wide range of geophysical situations. This issue is typically the drawback of the in-situ measurements. However, the co-locations will increase with time and we will extend such analysis to other buoys.

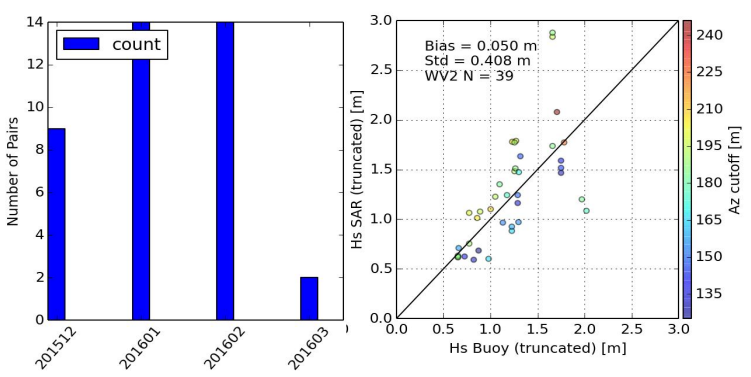

Illustration 7: Comparison between Stratus buoy and Sentinel-1 WV2. Left: Number of co-locations available at Stratus buoy per month. Right: Comparisons between effective significant wave height measured by Sentinel-1 A and measured by Stratus buoy. Effective significant wave height is evaluated for waves larger than the azimuth cut-off estimated from the Sentinel-1 products.

\section{Sentinel-1 A Level-4 product}

We applied the methodology to derive Level-4 from Sentinel-1 A observations. We partition Each 2D ocean wave spectrum into wave systems. This section provides an example of propagation obtained with Sentinel-1 A data acquired at the beginning of December 2015.

Figure 8 (a) and (b) show respectively an example of Sentinel-1 A acquisition and its corresponding ocean wave spectrum at location $97.07^{\circ} \mathrm{W}, 6.29^{\circ} \mathrm{E}$ the $10^{\text {th }}$ of December 2015 . This observation is from the orbit presented on Figure 8 (c), where blue dots stand for SAR acquisitions locations. On the image, the intensity modulation clearly shows a situation of cross seas with two swell systems. After partitioning, we can quality these two swell systems. One swell is from north west: Wavelength is $432.46 \mathrm{~m}$, Direction is $25^{\circ}$ from north and $\mathrm{Hs}$ is $1.58 \mathrm{~m}$ ). One swell is from south west (Wavelength is $376.75 \mathrm{~m}$ and Direction is $145^{\circ}$ from north and $\mathrm{Hs}$ is $1.37 \mathrm{~m}$ ). From these parameters, we propagate the swell backward and study the refocusing area as described in section 4 .

Figure 8 (c) shows the trajectories backward (red) as derived from 2D ocean wave measurements and obtained with one single orbit. The maximum propagation duration used here is 15 days. From these trajectories we can deduce two main areas from which the observations seem to be originated. One is in the north west part of the Pacific Ocean whereas the other one is in the south west part, two areas where extra-tropical storms takes place. When doing this exercise with all available observations we can locate in time and space the storm responsible for these two swell systems.

Figure 8 (d) shows the location of the storm related to the swell coming from north west and observed several thousands kilometers far away from its generation area. To find this refocusing area corresponding to a storm occurring the $29^{\text {th }}$ of November 2015, observations between the $30^{\text {th }}$ of November and the $10^{\text {th }}$ of December have been considered. This shows how the sampling of the swells propagating across the oceans is a key point of this method. As a consequence, any addition of extra observations is a real asset for such a product. It also shows that to be described by such a method, a storm need to generate waves that will be observed by satellites. Thus, the storm is fully characterized at posteriori when a minimum of observations has been done. Note also that depending on the storm location, the swell properties generated by this storm and the orbit characteristics of a given sensor, it can lead to non-favorable sampling of the swell. These situations will be reduced when several satellites will be used for such kind of analysis. 


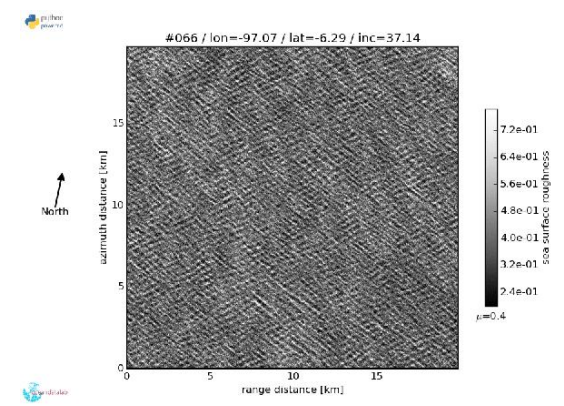

(a)

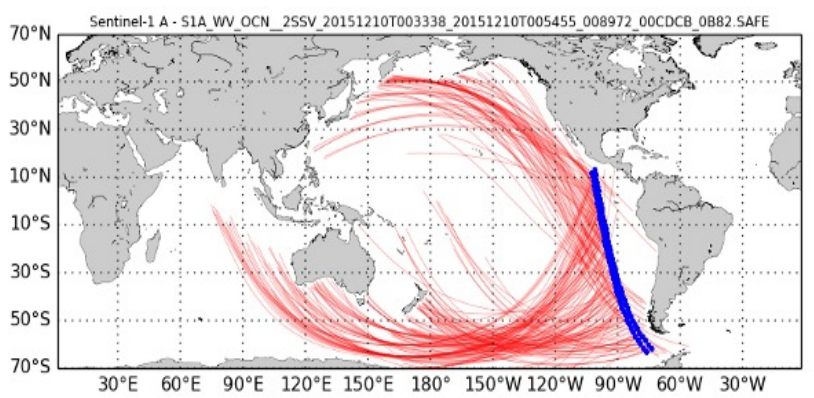

(c)

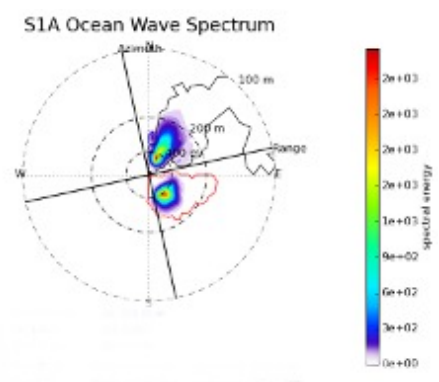

(b)

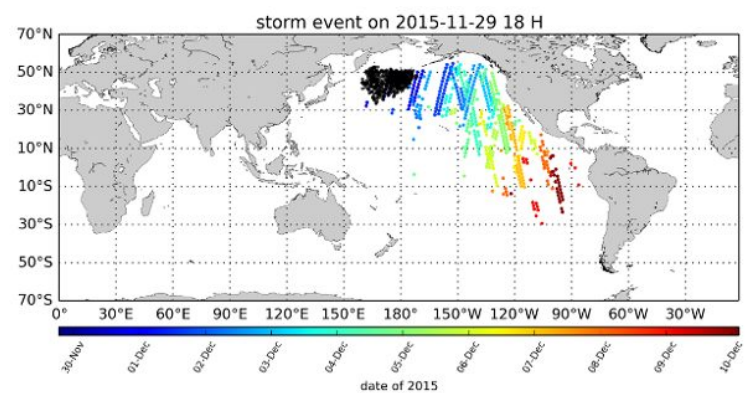

(d)

Illustration 8: (a) \& (b) Sentinel-1image and its associated ocean wave spectrum measurements for the $10^{\text {th }}$ of December 2015. Two swell systems can be observed. (c) Acquisitions corresponding to one orbit of Sentinel-1 A (blue dots) in South Pacific Ocean. Trajectories (red lines) of the swell partitions obtained after propagation backward during 15 days. (d) Example of refocusing area obtained for the $29^{\text {th }}$ of November 2015. This refocusing area corresponds to the storm that generated swell from north west observed on the acquisition presented on (a) \& (b).

Figure 9 (a)-(d), show the evolution of observations that have been associated to storms and propagated in time across the oceans as obtained from our analysis. Time steps presented here are 2015/12/02 at 23:30 UTC, 2015/12/03 at 23:30 UTC, 2015/12/06 at 11:30 UTC and 2015/12/08 at 11:30 UTC. Black circles indicate the refocusing areas related to storms. Black lines indicate the front of each wave systems. Pink crosses indicate the location of two buoys that are used for validation. Evolution of the swells originating from the 4 storms found the 2 th and the $4^{\text {th }}$ of December are well captured by the analysis. In particular, we can see the evolution of the swell fronts with respect to time with the longer (faster) swells propagating at the front.

For these the two buoys used for validation, Figure 9 (e) and (f) show the wavelength (period) obtained from the data analysis with respect to time between the $7^{\text {th }}$ of December 2015 and the $10^{\text {th }}$ of December 2015. As expected swells with the longest wavelengths are observed in the buoys vicinity at first. Thus, for a set of swell observations originating from the same storm and propagated at a given location though a certain time window, this time-period analysis systematically yields to a decrease of the wavelengths with respect to time. The same analysis is done at the buoys based on in-situ measurements for the same time range. Same decreases are observed. This shows that the period estimated from Sentinel-1 A, propagated and used to describe the waves at a storm scale are very consistent with the buoys measurements. This also shows how such kind of product can be used to produce information at a given location in time and space where no observations are available. In the case of inter-calibration exercises between different satellites, this is crucial to get data to compare with. When used in near-real time this kind of analysis also allow to forecast the swell arrival at a given location.

\section{Applications to the study of wind-generated waves by hurricanes}

The most mature application of such a combination of 2D ocean spectra swell measurements from space at an ocean basin scale (even if only measurements from one sensor were considered) is certainly the derivation of a new dissipation law for swell propagation across the ocean [6]. 
Here, we propose to investigate the capability of such data combination to check the consistency between the waves properties (wavelength, wave direction, significant wave height, spectral width) generated by storm events and the storm properties (intensity, extent, duration) in the particular cases of hurricanes. For now, we rely on Sentinel-1 A data as it is the only existing dataset available. The strength of this approach is to consider the data far from the source where the winds are expected to be less strong. Indeed, under severe conditions, SAR systems are not adapted to properly measure 2D ocean wave spectra.

We consider the case of hurricane Kilo (season 2015 in Pacific). Main properties of the hurricane are given by the best track file from NOAA. Trajectory, wind speed and hurricane speed are given on Figure 10. Based on a joint analysis between ocean wave spectra obtained from Sentinel-1 A and the location in space and time of the hurricane given by the track file, we study the wavelength distribution with respect to the center of the eye and hurricane characteristics such as hurricane wind speed and hurricane speed. In comparisons with the classical combination approach as described in the previous sub-section, the track file is used to help during the refocusing step.
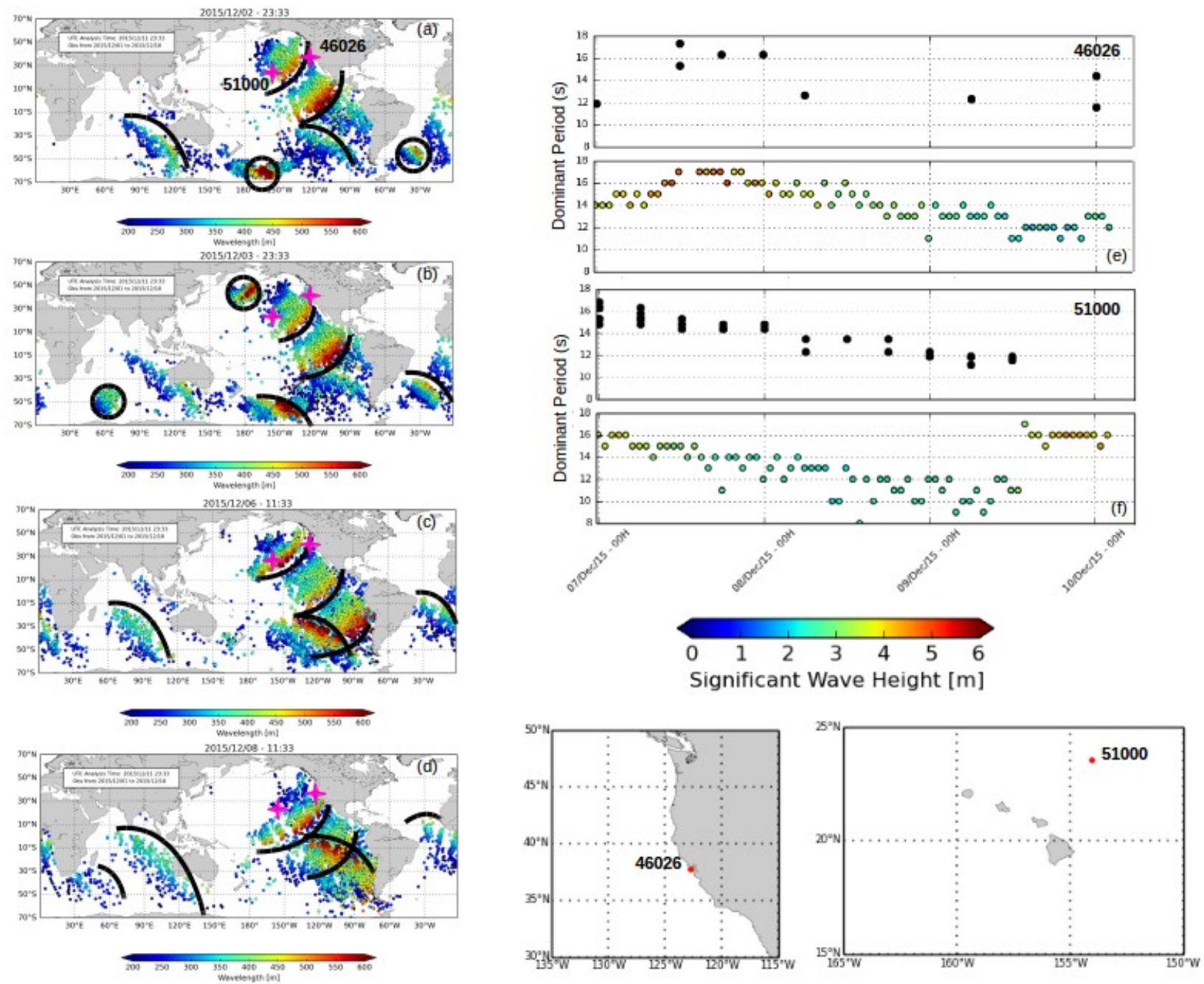

Illustration 9: (a-d) Example of propagation from the $2^{\text {nd }}$ to the $8^{\text {th }}$ of December 2015 for swell systems observed by Sentinel-1 A and associated to a storm. Black lines indicate swell fronts for a given swell ensemble belonging to one storm. Black circles indicate storm location. Pink crosses indicate the location of two NDBC buoys used for comparisons. (e-f) Time-period diagrams to monitor the arrival time of the swell at the two buoys with respect to the period. Time-period diagrams are produced for both data obtained from our analysis and in-situ measurements.

We consider two different moments where the hurricane properties are significantly different (see Figure 10). In the first case $\left(30^{\text {th }}\right.$ of August 2015), wind speed is larger than 100 knots and hurricane velocity is around $4 \mathrm{~m} / \mathrm{s}$. In the second 
case $\left(7^{\text {th }}\right.$ of September 2015), wind speed is around 80 knots whereas hurricane velocity remains the same (around 5 $\mathrm{m} / \mathrm{s}$ ). Figure 10 (bottom) presents the main output of such analysis. This is the wavelength distribution measured by Sentinel-1 A far from the source with respect to the hurricane heading. As obtained the distribution of wavelength generated by the hurricane is found to be elliptic.
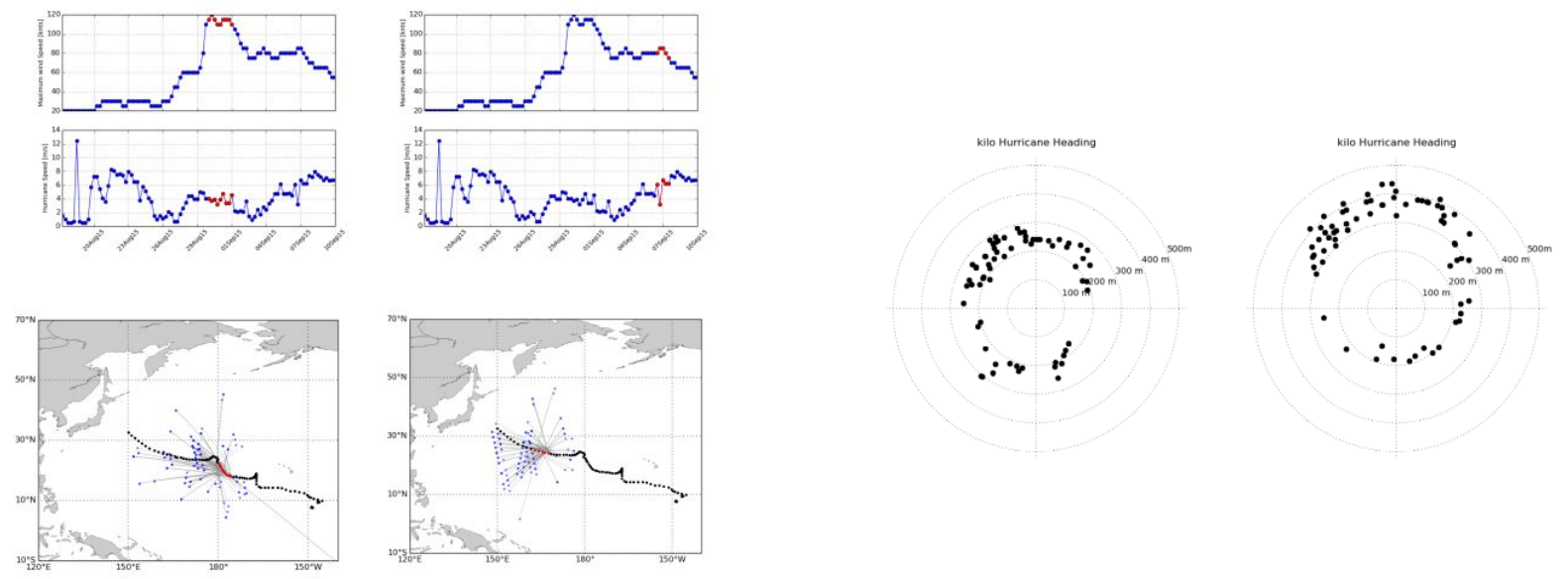

Illustration 10: Distribution of swell wavelength as measured from Sentinel-1 A with respect to hurricane heading for different hurricane conditions. Top-left panel: Kilo Hurricane wind speed and speed. Bottom-left panel: Kilo trajectory (black dots). Blue dots indicate the location of Sentinel-1 A observations corresponding to swell generated by the hurricane whose location is indicated by red dots. Right panel: Distribution of swell wavelength as measured from Sentinel-1 A with respect to hurricane heading.

In both situations, swell systems propagating ahead from the storm center have longer wavelengths than swell propagating in the opposite direction. As discussed by [9], this is due to the hurricane wind field asymmetry and its translation velocity. This induces different time durations for the wind forcing between the right and left sides of the hurricane center.

We also observe that the ellipticity of the wavelengths distribution is different for the two cases, meaning that the swell wavelengths generated by Kilo are not the same. In case 1, when the wind speed is maximum (>100 knts), we observe that swells have lower wavelengths than for case 2 when the wind speed is lower (around $80 \mathrm{knts}$ ). This is in agreement with the extended fetch concept. As explained by [9], the wave properties generated by the storm depends on the maximum wind speed of the hurricane but also the velocity of forward movement. Indeed, if the hurricane moves too slowly, the waves "outrun" the strong wind area and their wavelength stop increasing. If the hurricane move too rapidly waves are left behind and do not have time to growth.

Interestingly, our analysis could not retrieve the expected asymmetry between the right and left side of the hurricane. To date we have not explanation of this but investigations are going on and more cases need to be analyzed.

\section{CONCLUSIONS AND PERSPECTIVES}

In 2018, we should be in position of getting 2D ocean waves spectra measurements (Level-2 products) at global scale from 4 different satellites: the European satellites Sentinel-1 A (2014) and Sentinel-1 B (2016), the Chinese satellite GF3 (2016) and the Chinese/French CFOSAT/SWIM mission (2018). This would be a world first. Previous works based on ENVISAT/ASAR showed that such acquisitions could take benefit of this unique ability to measure wavelength, direction and significant wave height for each observed swell systems to gather all swell measurements belonging to one single storm event. It opened new area of applications such as dynamical validation (to increase the number of match-ups with in-situ buoy), the study of the space and time structure of long ocean swell fields [10], the investigation of the 
ability detect the long very long swell signature in ENVISAT/ASAR Wave Mode images [11] or the estimate of a new dissipation law for swell propagation across the ocean $[4,6]$.

Most of these applications could take benefit of a constellation of 4 different satellites. However, as the instruments does not have the same capacities, a careful validation and inter-calibration need to be done before any combination. We showed on Sentinel-1 A data how we can use the existing altimeters, available buoy networks and model outputs as reference to validate $2 \mathrm{D}$ ocean waves spectra measurements and monitor their performances during the mission lifetime. In particular, comparisons have to be performed by taking into account the fact that none of this missions (excepted from Sentinel-1 A \& B) will have the same range of validity (see table 1).

We presented a first example of application of the Fireworks concept to Sentinel-1 A data to combine the swell measurements as measured with this mission. Swells associated to storms and then propagated up to the coasts have been compared to two buoys from NDBC network. This dynamical co-location showed a good agreement between the arrival time of a given wavelength as obtained from SAR and measured by the buoy.

Finally, we introduced a new application consisting in using this kind of data to study the wavelength distribution (with respect to hurricane heading) of the wind-generated waves by a hurricane. In particular, we found that swell systems propagating ahead from the storm center have longer wavelengths than swell propagating in the opposite direction. We also showed that this asymmetry in the waves properties is related to the hurricane properties (here wind speed and hurricane velocity). This preliminary analysis confirms results from [9], showing that the distribution of wavelength is related to the hurricane properties (wind speed, hurricane velocity and size). However, more analysis and modeling need to be done to fully exploit such kind of data. For instance, we were not able to evidence the waves properties asymmetry between the right and left quadrant at the front of the hurricane.

*alexis.mouche@ifremer.fr; phone+33 (0) 298224929

\section{REFERENCES}

[1] V. Kerbaol, B. Chapron, P. W. Vachon, "Analysis of ERS-1/2 synthetic aperture radar wave mode imagettes.", J. Geophys. Res., 103(C4), 10.1029/97JC01579 (1998).

[2] Hasselmann, K., and S. Hasselmann, "On the nonlinear mapping of an ocean wave spectrum into a synthetic aperture radar image spectrum and its inversion.", Journal of Geophysical Research, 96(C6), 10.1029/91JC00302 (1991).

[3] Husson, R., [Development and validation of a global observation-based swell model using wave mode operating Synthetic Aperture Radar], PhD Thesis Université de Bretagne Occidentale, Brest (2012).

[4] F. Collard, F., F. Ardhuin, and B. Chapron, "Monitoring and analysis of ocean swell fields from space: New methods for routine observations.", J. Geophys. Res., 114, C07023, 10.1029/2008JC005215 (2009).

[5] D. Hauser, C. Tison, T. Amiot, L. Delayea, A. Mouche, G. Guitton, L. Aouf and P. Castillan, "CFOSAT: A new Chinese-French satellite for joint observations of ocean wind vector and directional spectra of ocean waves", This issue.

[6] F. Ardhuin, B. Chapron, and F. Collard, "Observation of swell dissipation across oceans.", Geophysical Research Letters, 36 (L06607), 10.1029/2008GL037030 (2009). 
[7] Munk, W. H., G. R. Miller, F. E. Snodgrass, and N. F. Barber, "Directional recording of swell from distant storms", Philosophical Transactions of the Royal Society of London. Series A, Mathematical and PhysicalSciences, 255 (1062), 505-584 (1963).

[8] Snodgrass, F. E., G. W. Groves, K. F. Hasselmann, G. R. Miller, W. H.Munk, and W. H. Powers, "Propagation of ocean swell across the Pacific", Philosophical Transactions of the Royal Society of London. Series A, Mathematical and Physical Sciences, 259 (1103), 431, (1966).

[9] Young I.R., [Wind Generated Ocean Waves], Elsevier (1999).

[10] M. T. Delpey, F. Ardhuin, F. Collard, and B. Chapron, "Space time structure of long ocean swell fields", J. Geophys. Res., 115, C12037, 10.1029/2009JC005885 (2010).

[11] R. Husson, F. Ardhuin, F. Collard, B. Chapron, and A. Balanche, "Revealing forerunners on Envisat's wave mode ASAR using the Global Seismic Network", Geophysical Research Letters, 39, L15609, doi:10.1029/2012GL052334 (2012). 